\title{
The Application of Wireless Sensor Networks in Management of Orchard
}

\author{
Guizhi Zhu \\ Institute of Sci-Tech Information, Guangdong Agricultural Academy of Sciences, 510640, \\ Guangzhou, China
}

\begin{abstract}
A monitoring system based on wireless sensor network is established, aiming at the difficulty of information acquisition in the orchard on the hill at present. The temperature and humidity sensors are deployed around fruit trees to gather the real-time environmental parameters, and the wireless communication modules with self-organized form, which transmit the data to a remote central server, can realize the function of monitoring. By setting the parameters of data intelligent analysis judgment, the information on remote diagnosis and decision support can be timely and effectively feed back to users.
\end{abstract}

Keywords: wireless sensor networks, orchard management, real time, database management system.

\section{Introduction}

Wireless Sensor Networks (WSN) is self-organized networks whose nodes are capable of sensing, gathering, processing and communicating data. Compared to the cable network can be several kilometers long, WSN don't depend on any preexisting infrastructure. So it can be deployed where it is unsafe or unwise for field studies. More recently, the availability of inexpensive sensors that are able to measure physical phenomena like temperature, pressure, light, humidity, or location of objects has enabled the development of WSN. WSN has drawn the attention of the research community since it arised in 1970, driven by a wealth of theoretical and practical challenges. Panchard.J(2008) compares two types of WSN architecture and apply them to india agriculture for guiding irrigating. An WSN application to real-world habitat monitoring was developed by Mainwaring et al(2002). Wang et al (2006) presents an overview on recent development of WSN and gives some examples of WSN applied in agriculture. Zhang W.Y et al(2007) proposes a coverage strategy to solve power management problem. Prabhat Ranjan proposes the possibility of monitoring parameters which impact the quality of life in rural india. Ian F. Akyildiz et al(2006) explores wireless multimedia sensor networks (WMSNs) architectures, and survey algorithms, protocols, hardware for WMSNs.

This paper develops an architecture of WSN and describes the components in detail. WSN is deployed in the orchard to fulfill these requirements: collecting the data 
information, such as temperature, light, soil moisture, humidity. Transmitting the data to a remote central server beyond the orchard. Developing a database management system running in the remote central server for data receiving and management. From the gathered information, the decision for orchard management can be made rightly.

\section{System Design}

We now describe the system architecture. It contains several components: sensor network, GPRS/INTERNET, remote central server, base station. Sensor nodes communicate with each other by self-organized format to make up sensor network, and transmit their data to the base station. The base station is responsible for transmitting data to remote central server through GPRS. A platform for orchard management system is established and runs in the remote central server. Finally, The data is displayed to users through a web-based user interface. Mobile devices, such as PDA and laptop, implement command to WSN through GPRS. The system architecture is as shown in Figure 1.

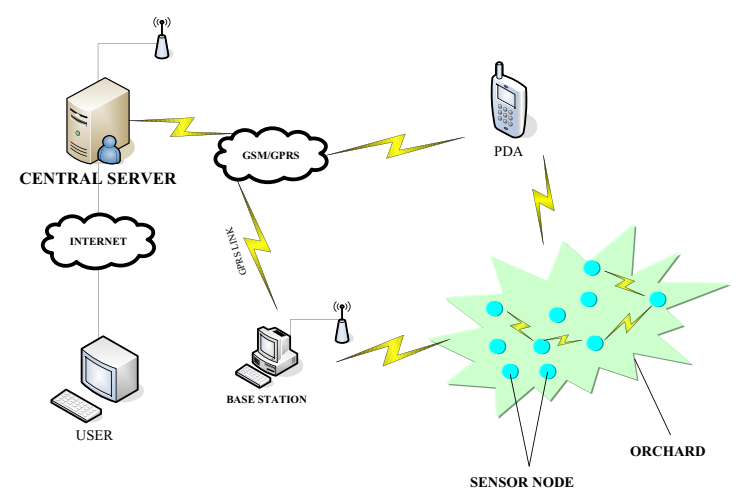

Fig. 1. System architecture

The operating system running in nodes is TinyOS, which is widely used. Power consumption is one of the main considered issues in the design of WSN. There is an efficient protocol used in WSN. We concentrate on low cost, low energy consumption sensors that will be densely deployed and provide detailed parameter information in the surrounding environment of orchard.

The Web-based Data Management plays an important role and is responsible for storing all information in WSN, such as sensor location, data received from a particular node. The remote central server stores and displays data to users, also, one can acquire and send commands to the WSN, thus enhance interaction with the environment.

Scientific users hope to enable widespread environmental monitoring and collection of experimental data. 


\section{Deployment and Orchard Management}

We proceeded to the deployment of WSN in agricultural base in 2008, which is a high-tech agricultural base of the Institute of Sci-Tech Information, Guangdong Agricultural Academy of Sciences. The agricultural base is an area of 700 acres of hilly areas. Fixed telephone and power supply is available. There is a radio relay station in the north-west of the base, so communication signals are very well.

We deployed 20 different sensors among orchard to collecting the data information, such as temperature, light, soil moisture, humidity. Zigbee technology forms connections between the nodes. The platform used is MICA2, which was produced by Crossbow. The nodes are generally stationary after deployment except for a few mobile nodes. The sensors collect data and transmit data every 5 minutes. Our main goals are prolonging the life of the network and preventing connectivity degradation through aggressive energy management as the batteries cannot usually be replaced because of operations in hostile or remote environments. Dynamic environmental conditions requiring the system is adaptive to changing connectivity and node failure.

A database management system is developed running in the remote central server for data receiving and management. From the gathered information, the decision for orchard management can be made rightly by users. A map of the deployment in orchard is depicted in Figure 2.

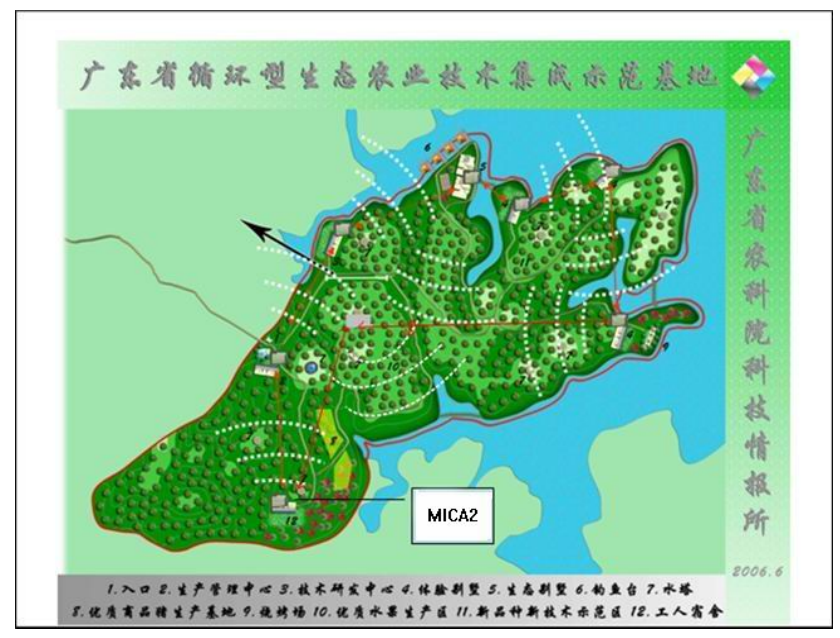

Fig. 2. Orchard deployment

\section{Discussion}

Applications of WSN in agriculture are still at its early development stage. This paper has made a description of WSN, the system has been used in orchard, running in an automated fashion. Deployment issues are covered in detail. At present, the system hasn't be used widely. It's important to improve the energy efficiency and reduce the cost of sensors. 
In our deployment, sensors and central server is equipped with a GPRS unit, the system achieves remote monitoring by WSN and the GPRS/INTERNET, which overcomes the limitations of traditional on-site closed monitoring. The future development direction of the system is develop the decision-support system.

\section{Acknowledgements}

This work was supported by a grant from Guangdong Natural Science Foundation (8151064001000009), Guangdong Provincial Public Laboratory on Wild Animal Conservation, Management, Key Technologies R \& D Program of Guangdong Province of China (2008A020100026, 2007A020300004-7).

\section{References}

Mainwaring, A., Polastre, J., Szewczyk, R., Culler, D., Anderson, J.: Wireless Sensor Networks for Habitat Monitoring

Akyildiz, I.F., et al.: A survey on wireless multimedia sensor networks. Computer networks, 140 (2006)

Panchard, J.: Wireless Sensor Networks for Marginal Farming in India, PhD Thesis (2008)

Ranjan, P.: Sensor networks to monitor quality of life in rural area, http://intranet.daiict.ac.in/ ranjan/research/papers/ nsc_93rd_talk.pdf

Wang, N., et al.: Wireless sensors in agriculture and food industry-Recent development and future perspective. Computers and electronics in agriculture (2006)

Zhang, W., et al.: Energy-efficient coverage stategy in wireless sensor networks based on clustering by k-coverage. In: Proceedings of the 4th information technology in agriculture (ISIITA), Beijing,China, October 26-29, pp. 546-550 (2007) 